\title{
THE $H^{2}$ CORONA PROBLEM AND $\bar{\partial}_{b}$ IN WEAKLY PSEUDOCONVEX DOMAINS
}

\author{
MATS ANDERSSON
}

\begin{abstract}
We derive a Bochner-Kodaira-Nakano-Morrey-Kohn-Hörmander type equality in holomorphic vector bundles and obtain $L^{2}$-estimates for $\bar{\partial}_{b}$ in a pseudoconvex domain that admits a plurisubharmonic $C^{2}$ defining function. We combine these with the trick in Wolff's proof of the corona theorem and obtain a $H^{2}$-corona theorem in such a domain.
\end{abstract}

\section{INTRODUCTION}

Let $D$ be a domain in $\mathbb{C}^{n}$ and $g_{i} \in H^{\infty}(D)$ such that

$$
\sum_{1}^{k}\left|g_{i}\right|^{2} \geq \delta^{2}>0 \text {. }
$$

The problem whether there are $u_{j} \in H^{\infty}(D)$ such that $\sum_{1}^{k} g_{i} u_{i}=1$ is known as the corona problem. The answer is affirmative in e.g. all finitely connected domains in $\mathbb{C}$, but unknown even in the ball if $n>1$. In [11 and 5] are constructed smooth domains in $\mathbb{C}^{3}$ and $\mathbb{C}^{2}$ which have strictly pseudoconvex boundary in all but one point, but in which the corona theorem fails. However it follows from [13] that in case of two generators, i.e. $k=2$, in a strictly pseudoconvex domain there is a solution in BMO.

It is clear that if the corona problem is solvable, then to any $q \in H^{2}(D)$ there are $u_{j} \in H^{2}(D)$ such that $\sum g_{j} u_{j}=q$. In this paper we prove such a theorem in a pseudoconvex domain $D$ admitting a $C^{2}$ plurisubharmonic defining function $\rho$, i.e. $\rho$ be of class $C^{2}$ in a neighborhood of $\bar{D}, D=\{\rho<0\}, d \rho \neq 0$ on $\partial D$ and $i \partial \bar{\partial} \rho \geq 0$ in $D$. In particular, the domains in [11 and 5] are of this kind. However, there are examples of pseudoconvex $C^{2}$ domains without plurisubharmonic defining function, see [3].

Theorem 1. Suppose $D$ is a pseudoconvex domain in $\mathbb{C}^{n}$ with a $C^{2}$ plurisubharmonic defining function. Let $g$ be a $j \times k$-matrix of functions in $H^{\infty}(D)$ such that

$$
\operatorname{det} g g^{*} \geq \delta^{2}>0 .
$$

Received by the editors November 27, 1990 and, in revised form, January 17, 1992. 1991 Mathematics Subject Classification. Primary 32A10, 32A35, 32F15, 32F20.

Key words and phrases. Corona theorem, $\bar{\partial}_{b}$-equation. 
Then to every j-column $q$ of functions in $H^{2}(D)$ there is a $k$-column $u$ in $H^{2}(D)$ such that

$$
g u=q
$$

and $\|u\|_{H^{2}} \leq C_{\delta}\|q\|_{H^{2}}$. If $j=1$ one can take $C_{\delta}=C_{\varepsilon} / \delta^{1+\varepsilon+\min (n, k-1)}$ where $C_{\varepsilon}$ only depends on $\|g\|_{\infty}, n, k$ and $\varepsilon>0$.

Note that (2) implies that $g$ is surjective and hence that (3) is pointwise solvable. In fact it is enough to assume that $g$ has constant rank, the product of the nonzero eigenvalues of $g g^{*}$ are bounded by $\delta^{2}$ from below and that (3) is pointwise solvable to get the conclusion of Theorem 1, cf. [1].

Since any smooth pluriharmonic $\chi$ on $\bar{D}$ has the form $\chi=\log |f|^{2}$ for some nonvanishing holomorphic $f$ (at least if $H^{1}(\bar{D}, \mathbb{C})=0$ ), we can apply Theorem 1 to $q / f$ instead of $q$ and obtain a solution to (3) such that

$$
\int_{\partial D}|u|^{2} e^{-\chi} \leq c_{\delta}^{2} \int_{\partial D}|q|^{2} e^{-\chi} .
$$

If $n=1, \chi$ can be freely chosen in (4) and this implies, see [2] or [1], that there is a bounded solution $u$ such that $\|u\|_{\infty} \leq c_{\delta}\|q\|_{\infty}$ if $q$ is bounded. Hence Theorem 1 is equivalent to the corona theorem in the unit disc.

Theorem $\mathbf{1}^{\prime}$. Let $D$ be as in Theorem 1 . If $q$ and $g=\left(g_{1}, \ldots, g_{k}\right)$ in $H^{\infty}(D)$ satisfy

$$
|q| \leq|g|^{1+\varepsilon+\min (n, k-1)},
$$

then there is a solution $u$ to $(3)$ in $H^{2}(D)$ such that

$$
\int_{\partial D}|u|^{2} e^{-\chi} d S \leq C_{\varepsilon} \int_{\partial D} e^{-\chi} d S .
$$

Again, for $n=1$, this implies that there is a bounded solution if $|q| \leq|g|^{2+\varepsilon}$. This also follows from Wolff's proof of the corona theorem, see [7].

Corollary. If $q=q_{1} q_{2}$ where $q_{1}$ satisfies (5) and $q_{2} \in H^{2}(D)$ is nonvanishing, then there is a solution $u$ to (3) such that

$$
\int_{\partial D}|u|^{2} d S \leq C_{\varepsilon} \int_{\partial D}\left|q_{2}\right|^{2} d S .
$$

Our proof of Theorem 1 (and $1^{\prime}$ ), as most proofs of the corona theorem, is based on an estimate of solutions of a $\bar{\partial}_{b}$-equation. This approach was first introduced by Hörmander in [10]. Using the Koszul complex one can, in principle, reduce the theorem to systems of (scalar-valued) $\bar{\partial}$ - (or $\bar{\partial}_{b}$-) equations, at least if $j=1$. However, unless $n=1$ or $k \leq 2$, one has to solve, successively, a sequence of $\bar{\partial}$-equations (involving forms of higher bidegree) and this seems to lead to considerable difficulties. Instead we reformulate the theorem as a $\bar{\partial}_{b}$-problem in a holomorphic vector bundle, following the lines in [12] (but with $\bar{\partial}$ replaced by $\bar{\partial}_{b}$ ), in which $L^{2}$-estimates for division problems are treated in a very general setting.

The $\bar{\partial}_{b}$-equation is treated by a generalization of a variant due to Berndtsson [2], of the Morrey-Kohn-Hörmander identity (see $\S 3$ ). In [2], this new identity was used to get $L^{2}(\partial D)$-estimates for the (scalar-valued) $\bar{\partial}_{b}$-equation. Since we 
need estimates for $\bar{\partial}_{b}$ in a vector bundle, our first aim is to generalize to this case. This leads to a $L^{2}(\partial D)$-theorem for $\bar{\partial}_{b}$ in a holomorphic vector bundle over a pseudoconvex domain in a Kähler manifold, see Theorem 2 in $\S 4$.

However, in order to prove Theorem 1 (and Theorem $1^{\prime}$ ) we cannot use Theorem 2 directly since it just deals with a size estimate of the right-hand side. One also has to take into account an appropriate estimate of derivatives. This is the trick introduced by Wolff in his proof of the corona theorem, see [7].

The paper is divided into six sections. After some necessary preliminaries in $\S 1$, we discuss the $\bar{\partial}_{b}$-equation in $\S 2$. In $\S 3$ we derive the above-mentioned equality and show its connection to the Morrey-Kohn-Hörmander equality as well as the Bochner-Kodaira-Nakano equality. In $\S 4$ we prove our $L^{2}$-estimate for $\bar{\partial}_{b}$ (Theorem 2) and in the remaining two sections we prove Theorem 1 and Theorem $1^{\prime}$.

\section{NOTATIONAL PRELIMINARIES}

Let $X$ be a Kähler manifold with fundamental form $\omega$, so that $d V=\omega^{n} / n$ ! is the volume measure on $X$, and let $D=\{\rho<0\}$ be a relatively compact domain in $X$, where $\rho$ is smooth and $d \rho \neq 0$ on $\partial D$. We give $\partial D$ the orientation so that a $(2 n-1)$-form $\alpha$ is oriented on $\partial D$ if and only if $d \rho \wedge \alpha$ is oriented on $X$. Then the surface measure $d S$ on $\partial D$ is given by $d S=$ $d \rho /|d \rho|\lrcorner d V$ on $\partial D$, where inner multiplication $\lrcorner$ for forms $\alpha$ and $\beta$ is defined by

$$
\langle\alpha\lrcorner \beta, \gamma\rangle=\langle\beta, \bar{\alpha} \wedge \gamma\rangle .
$$

Here $\langle$,$\rangle is the induced inner product for forms, i.e. if *$ is the complex-linear Hodge star operator, then $\langle\alpha, \beta\rangle d V=*(\alpha \wedge * \bar{\beta})$. Note that $* *=(-1)^{p+q}$ on $(p, q)$-forms (since $X$ has even real dimension) and that $\alpha\lrcorner \beta=*(\alpha \wedge * \beta)$ for any 1 -form $\alpha$. If $\alpha$ is a $(2 n-1)$-form, we have that

$$
\int_{\partial D} \alpha=\int_{\partial D} *(d \rho \wedge \alpha) d S /|d \rho| .
$$

To see (2), notice that $d \rho \wedge *(d \rho \wedge \alpha) d S /|d \rho|=*(d \rho \wedge \alpha) d \rho \wedge d S /|d \rho|=$ $*(d \rho \wedge \alpha) d V=d \rho \wedge \alpha$, and hence both integrands in (2) are equal, considered as forms on $\partial D$.

If $E \rightarrow X$ is a holomorphic vector bundle over $X$ with hermitian metric $\langle,\rangle_{E}$, then we get a metric for $\xi, \eta \in \mathscr{E}_{p, q}(X, E) \quad\left(=\mathscr{E}\left(X, \Lambda^{p, q} T^{*} \otimes E\right)\right.$ i.e. smooth $E$-valued $(p, q)$-forms) by putting $\left\langle\alpha \cdot s, \alpha^{\prime} \cdot s^{\prime}\right\rangle=\left\langle\alpha, \alpha^{\prime}\right\rangle\left\langle s, s^{\prime}\right\rangle_{E}$ on composite elements. If $s \rightarrow s^{\prime}$ is the conjugate linear mapping from $E$ to its dual bundle $E^{*}$, such that $s^{\prime} \cdot s=\langle s, s\rangle_{E}$, and $\bar{*}: \mathscr{E}_{p, q}(X, E) \rightarrow \mathscr{E}_{q, p}\left(X, E^{*}\right)$ by putting $\bar{*}(\alpha \cdot s)=* \bar{\alpha} \cdot s^{\prime}$ on composite elements, then

$$
\langle\xi, \eta\rangle d V=\xi \wedge \bar{*} \eta, \quad \xi, \eta \in \mathscr{E}_{p, q}(X, E) .
$$

Moreover, $\alpha\lrcorner \xi=\bar{*}(\bar{\alpha} \wedge \bar{*} \xi)$, cf. (5), if $\alpha$ is a 1-form and $\xi$ any $E$-valued form.

For $\xi, \eta \in \mathscr{E}_{p, q}(X, E)$, one of which has compact support, we have the inner product

$$
(\xi, \eta)=f\langle\xi, \eta\rangle d V=\int \xi \wedge \bar{*} \eta .
$$


Let $D=D^{\prime}+D^{\prime \prime}$ denote the Chern connection on $E$ as well as on $E^{*}$ (with respect to $\left.\langle,\rangle_{E}\right)$. Then the formal adjoints $\left(D^{\prime}\right)^{*}$ and $\left(D^{\prime \prime}\right)^{*}$ are given by $\left(D^{\prime \prime}\right)^{*}=-\bar{*} D^{\prime \prime} \bar{*},\left(D^{\prime}\right)^{*}=-\bar{*} D^{\prime} \bar{*}$.

If $s$ is a holomorphic section to $E$ then $D^{\prime \prime}(\alpha \cdot s)=(\bar{\partial} \alpha) \cdot s$ and therefore we sometimes write $\bar{\partial}$ instead of $D^{\prime \prime}$.

\section{THE $\bar{\partial}_{b}$-EQUATION}

Using the notation from $\S 1$ we have

Proposition 1. Suppose $\eta \in \mathscr{E}_{p, q+1}(\bar{D}, E)$ and $\xi \in \mathscr{E}_{p, q}(\bar{D}, E)$. Then

$$
\left.\int_{D}\left\langle D^{\prime \prime} \xi, \eta\right\rangle d V-\int_{D}\left\langle\xi,\left(D^{\prime \prime}\right)^{*} \eta\right\rangle d V=\int_{\partial D}\langle\xi, \partial \rho\lrcorner \eta\right\rangle d S /|d \rho| .
$$

Remark. Throughout this paper $\left(D^{\prime \prime}\right)^{*}$ denotes the formal adjoint of $D^{\prime \prime}$. When dealing with the $D^{\prime \prime}$-Neumann problem $\left(D^{\prime \prime}\right)^{*}$ is an operator with a specified domain $\operatorname{dom}\left(D^{\prime \prime}\right)^{*}$. For instance, (1) implies that $\eta \in \mathscr{E}_{p, q}(\bar{D}, E)$ is in $\operatorname{dom}\left(D^{\prime \prime}\right)^{*}$ if and only if $\left.\partial \rho\right\lrcorner\left.\eta\right|_{\partial D}=0$.

Sketch of proof. By (1), (3), and (2) in $\S 1$ we have that

$$
\left.\int_{\partial D}\langle\xi, \partial \rho\lrcorner \eta\right\rangle d S /|d \rho|=\int_{\partial D} *(\bar{\partial} \rho \wedge \xi \wedge \bar{*} \eta) d S /|d \rho|=\int_{\partial D} \xi \wedge \bar{*} \eta,
$$

so by Stokes' theorem and bidegree reasons the right-hand side of (1) equals $\int_{D} d(\xi \wedge \bar{*} \eta)=\left(D^{\prime \prime} \xi, \eta\right)-\left(\xi,\left(D^{\prime \prime}\right)^{*} \eta\right)$.

Let $f \in \mathscr{E}_{n, q+1}(\bar{D}, E)$. We say that an $E$-valued form (current) $u$ on $\partial D$ solves $\bar{\partial}_{b} u=f$ if

$$
\left.\int_{\partial D}\langle u, \partial \rho\lrcorner \alpha\right\rangle d S /|d \rho|=\int_{D}\langle f, \alpha\rangle d V
$$

for all $\alpha \in \mathscr{E}_{n, q+1}(\bar{D}, E)$ such that $\left(D^{\prime \prime}\right)^{*} \alpha=0$.

Hence, by (1), $u$ must have bidegree $(n, q)$ in the sense that $\alpha \wedge \bar{\partial} \rho$ has bidegree $(n, q+1)$, and $\bar{\partial} f=0$ (since one can take $\alpha=\bar{*} \bar{\psi}$ for any $\bar{\partial}$-closed $\psi)$. Notice that

$$
\|u\|_{\partial D}^{2}=\int_{\partial D}|u \wedge \bar{\partial} \rho|^{2} d S /|\partial \rho|_{3}
$$

defines a norm for the space of smooth $(n, q)$-forms on $\partial D$, and let $L_{n, q}^{2}(\partial D, E)$ be its completion with respect to this norm.

Remark. To be more precise, any $u \in \mathscr{E}\left(\partial D, \Lambda^{n, q} T(X) \otimes E\right)$ has an orthogonal decomposition $u=u_{1}+u_{2}$, where $\bar{\partial} \rho \wedge u_{1}=0$ and $\left.\bar{\partial} \rho\right\lrcorner u_{2}=0$. Thus $(n, q)$ forms (currents) $V$ such that $\partial \rho\lrcorner V=0$ can be isometrically identified with intrinsic $(n, q)$-forms (currents) on $\partial D$.

Proposition 2. Let $f \in \mathscr{E}_{n, q+1}(\bar{D}, E)$ be $D^{\prime \prime}$-closed. Then $\bar{\partial}_{b} u=f$ has a solution in $L_{n, q}^{2}(\partial D, E)$ with norm $C$ if and only if

$$
\left.\left|\int_{D}\langle f, \alpha\rangle d V\right|^{2} \leq C^{2} \int_{\partial D} \mid \partial \rho\right\lrcorner\left.\alpha\right|^{2} d S /|d \rho|^{2}
$$

for all $\left(D^{\prime \prime}\right)^{*}$-closed $\alpha \in \mathscr{E}_{n, q+1}(\bar{D}, E)$. 
Proof. First suppose there is such a solution $u$ considered as a current such that $\bar{\partial} \rho \wedge u=0$, cf., the remark above. Then $\left.\int_{D}\langle f, \alpha\rangle d V=\int_{\partial D}\langle u, \partial \rho\lrcorner \alpha\right\rangle d S /|d \rho|$ so by Schwarz inequality,

$$
\left.\left|\int_{D}\langle f, \alpha\rangle d V\right|^{2} \leq \int_{\partial D}|u|^{2} d S /|d \rho| \int_{\partial D} \mid \partial \rho\right\lrcorner\left.\alpha\right|^{2} d S /|d \rho|
$$

which implies (4). For the converse, assume that (4) holds. Define the linear functional $\Lambda(\partial \rho\lrcorner \alpha)=\int_{D}\langle f, \alpha\rangle d V$ on $E$-valued $(n, q)$-forms on $\partial D$ of the form $\partial \rho\lrcorner \alpha$ where $\left(D^{\prime \prime}\right)^{*} \alpha=0$ in $D$. By (4) it is well defined and $L^{2}$ bounded, so there is a $u$ such that $\int_{\partial D}|u|^{2} /|d \rho| d S \leq C^{2}$ and (2) holds.

In the next paragraph we shall derive an equality which gives a possibility to obtain estimates like (4).

\section{A BOCHNER-KodAIRA-NAKANO-MORREY-KoHN-HöRMANDER TYPE EQUALITY}

In the notation from $\S 1$, the Bochner-Kodaira-Nokano identity is

$$
\left(D^{\prime \prime}\right)^{*} D^{\prime \prime}+D^{\prime \prime}\left(D^{\prime \prime}\right)^{*}=\left(D^{\prime}\right)^{*} D^{\prime}+D^{\prime}\left(D^{\prime}\right)^{*}+i[\boldsymbol{\Theta}, \Lambda]
$$

where $\Lambda$ is inner multiplication with the fundamental form $\omega, \Theta$ is the curvature tensor on $E$, i.e. $\Theta=D^{2}$, and [, ] denotes commutator.

If $X$ is compact (so that no boundary terms occur) (1) implies the estimate $\left\|D^{\prime \prime} \xi\right\|^{2}+\left\|\left(D^{\prime \prime}\right)^{*} \xi\right\|^{2} \geq(i[\Theta, \Lambda] \xi, \xi)$. If $a=i[\Theta, \Lambda]$ happens to be nonnegative on $E$-valued $(p, q)$-forms and $f$ is a $D^{\prime \prime}$-closed $(p, q)$-form one gets [also using a local regularity result for the elliptic operator $\left.\bar{\square}=D^{\prime \prime}\left(D^{\prime \prime}\right)^{*}+\left(D^{\prime \prime}\right)^{*} D^{\prime \prime}\right]$ the estimate

$$
\left|\int\langle f, \xi\rangle d V\right|^{2} \leq \int\left\langle a^{-1} f, f\right\rangle d V \int\left|\left(D^{\prime \prime}\right)^{*} \xi\right|^{2} d V \text { for all } \xi \in \mathscr{E}_{p, q}(X, E),
$$

which means that there is a solution to $D^{\prime \prime} u=f$ with $\|u\|^{2} \leq \int\left(a^{-1} f, f\right) d V$, provided the right-hand side is finite.

In a domain $D$ with boundary, one leads to study the $D^{\prime \prime}$-Neumann problem and here the starting point is the Morrey-Kohn-Hörmander identity. We will derive it below from (1). To deal with the $\bar{\partial}_{b}$-equation on $\bar{D}$ we need still another equality (Proposition 7) first found and used in [2] in the case of $(0,1)$ forms (see the remark below) and trivial bundle. This one too will be derived from (1).

We first note how the various geometrical objects are affected if our original metric $\langle$,$\rangle on E$ is modified.

Proposition 3. If $\langle$,$\rangle is changed to \langle\rangle e^{-\varphi}$, then by obvious use of the index $\varphi$,

$$
\begin{aligned}
& \left.\left(D^{\prime \prime}\right)^{* \varphi}=\left(D^{\prime \prime}\right)^{*}+\partial \varphi\right\lrcorner, \\
& D_{\varphi}^{\prime}=D^{\prime}-\partial \varphi \wedge \\
& \boldsymbol{\Theta}_{\varphi}=\boldsymbol{\Theta}+\partial \bar{\partial} \varphi
\end{aligned}
$$

and

$$
\left(D_{\varphi}^{\prime}\right)^{* \varphi}=\left(D^{\prime}\right)^{*}
$$


Any of these follows from well-known identities, see e.g. [8], or by simple computations.

Now put $\varphi=t \log (-1 / \rho)$ in $D=\{\rho<0\}$ so that $\exp (-\varphi)=(-\rho)^{t}$ and $\partial \varphi=O(-1 / \rho)$. Also put $(,)_{\varphi}=\int_{D}\langle,\rangle e^{-\varphi} d V$. If $t>2$, we can, cf. $(2), \ldots,(5)$, integrate by parts and obtain

$$
\left\|D^{\prime \prime} \alpha\right\|_{\varphi}^{2}+\left\|\left(D^{\prime \prime}\right)^{* \varphi} \alpha\right\|_{\varphi}^{2}=\left\|D_{\varphi}^{\prime} \alpha\right\|_{\varphi}^{2}+\left\|\left(D_{\varphi}^{\prime}\right)^{* \varphi} \alpha\right\|_{\varphi}^{2}+i\left(\left[\boldsymbol{\Theta}_{\varphi}, \Lambda\right] \alpha, \alpha\right)_{\varphi}
$$

from (1). Our next task is to compute the various terms in (6). We assume that $\alpha \in \mathscr{E}_{n, q}(\bar{D}, E)$ so that $D_{\varphi}^{\prime} \alpha=0$ and $\left[\Theta_{\varphi}, \Lambda\right] \alpha=\Theta_{\varphi} \Lambda \alpha$. Since, by (2),

$$
\left.\left.\left(D^{\prime \prime}\right)^{* \varphi}=\left(D^{\prime \prime}\right)^{*}+\partial \varphi\right\lrcorner=\left(D^{\prime \prime}\right)^{*}-t(\partial \rho / \rho)\right\lrcorner
$$

we get

$$
\begin{aligned}
& \left\|\left(D^{\prime \prime}\right)^{* \varphi} \alpha\right\|_{\varphi}^{2}=\int_{D}(-\rho)^{t}\left|\left(D^{\prime \prime}\right)^{*} \alpha\right|^{2} d V \\
& \left.\left.\quad+2 t \operatorname{Re} \int_{D}(-\rho)^{t-1}\left\langle\left(D^{\prime \prime}\right)^{*} \alpha, \partial \rho\right\lrcorner \alpha\right\rangle d V+t^{2} \int_{D}(-\rho)^{t-2} \mid \partial \rho\right\lrcorner\left.\alpha\right|^{2} d V .
\end{aligned}
$$

By (4),

$$
\boldsymbol{\Theta}_{\varphi}=\boldsymbol{\theta}-t \partial \bar{\partial} \rho / \rho+t \partial \rho \wedge \bar{\partial} \rho / \rho^{2}
$$

We need also

Lemma 4. If $\alpha \in \mathscr{E}_{n, q}(\bar{D}, E)$, then

$$
i\langle\partial \rho \wedge \bar{\partial} \rho \wedge \Lambda \alpha, \alpha\rangle=\mid \partial \rho\lrcorner\left.\alpha\right|^{2} .
$$

Lemma 5. If $\psi \in \mathscr{E}(\bar{D})$, then

$$
\int_{D}(-\rho)^{t-1} \psi d V \rightarrow \int_{\partial D} \psi d S /|d \rho|
$$

when $t \searrow 0$.

Taking these for granted for the moment we get from (6), (7), (8) and (9) that

$$
\begin{aligned}
\int_{D}(-\rho)^{t}\left|D^{\prime \prime} \alpha\right|^{2} d V+\int_{D}(-\rho)^{t}\left|\left(D^{\prime \prime}\right)^{*} \alpha\right|^{2} d V \\
\left.\quad+2 t \operatorname{Re} \int_{D}(-\rho)^{t-1}\left\langle\left(D^{\prime \prime}\right)^{*} \alpha, \partial \rho\right\lrcorner \alpha\right\rangle d V \\
\left.\quad+t^{2} \int_{D}(-\rho)^{t-2} \mid \partial \rho\right\lrcorner\left.\alpha\right|^{2} d V \\
=\int_{D}(-\rho)^{t}\left|\left(D^{\prime}\right)^{*} \alpha\right|^{2} d V+\int_{D}(-\rho)^{t} i\langle\Theta \Lambda \alpha, \alpha\rangle d V \\
\left.\quad+t \int_{D}(-\rho)^{t-1}\langle i \partial \bar{\partial} \rho \wedge \Lambda \alpha, \alpha\rangle d V+t \int_{D}(-\rho)^{t-2} \mid \partial \rho\right\lrcorner\left.\alpha\right|^{2} d V
\end{aligned}
$$

for $t>2$ and $\alpha \in \mathscr{E}_{n, q}(\bar{D}, E)$.

If we now assume that $D^{\prime \prime} \alpha=\left(D^{\prime \prime}\right)^{*} \alpha=0$, combine the last terms on each side of equality (10) and let $t \searrow 1$ we get 
Proposition 6. Suppose $\alpha \in \mathscr{E}_{n, q}(\bar{D}, E)$ and $D^{\prime \prime} \alpha=\left(D^{\prime \prime}\right)^{*} \alpha=0$. Then

$$
\begin{aligned}
& i \int_{D}(-\rho)\langle\Theta \Lambda \alpha, \alpha\rangle d V+i \int_{D}\langle\partial \bar{\partial} \rho \Lambda \alpha, \alpha\rangle d V \\
& \left.\quad+\int_{D}(-\rho)\left|\left(D^{\prime}\right)^{*} \alpha\right|^{2} d V=\int_{\partial D} \mid \partial \rho\right\lrcorner\left.\alpha\right|^{2} d S /|d \rho| .
\end{aligned}
$$

Remark. Suppose $D \subset \mathbb{C}^{n}$. If $\langle$,$\rangle is the metric e^{-\psi}$ on the trivial line bundle (so that $\boldsymbol{\Theta}=\partial \bar{\partial} \psi)$ and $(n, 1)$-forms are identified with $(0,1)$-forms in the obvious way, then (11) is exactly Proposition 5 in [2].

In a similar way we can also obtain the Morrey-Kohn-Hörmander identity.

Proposition 7. If $\alpha \in \mathscr{E}_{n, q}(\bar{D}, E)$ and $\left.\partial \rho\right\lrcorner\left.\alpha\right|_{\partial D}=0$, then

$$
\begin{aligned}
& \int_{D}\left|D^{\prime \prime} \alpha\right|^{2} d V+\int_{D}\left|\left(D^{\prime \prime}\right)^{*} \alpha\right|^{2} d V=\int_{D}\left|\left(D^{\prime}\right)^{*} \alpha\right|^{2} d V \\
& \quad+i \int_{\partial D}\langle\partial \bar{\partial} \rho \Lambda \alpha, \alpha\rangle d S /|d \rho|+i \int_{D}\langle\Theta \Lambda \alpha, \alpha\rangle d V .
\end{aligned}
$$

Proof. By assumption $\partial \rho\lrcorner \alpha=O(-\rho)$ so (12) follows from (10) when $t \searrow$ 0 .

We conclude this paragraph with proofs of the lemmas.

Proof of Lemma 4. Fix a point and $(1,0)$-forms $\omega_{1}, \ldots, \omega_{n}$ such that $\omega=$ $\sum \omega_{j} \wedge \bar{\omega}_{j}$ and $\partial \rho=\omega_{1}$ at this point. We can write $\alpha=\alpha^{\prime}+\alpha^{\prime \prime}=\omega_{1} \wedge \bar{\omega}_{1} \wedge$ $\gamma+\alpha^{\prime \prime}$, such that $\gamma$ and $\alpha^{\prime \prime}$ do not contain $\bar{\omega}_{1}$. Then $\omega_{1} \wedge \bar{\omega}_{1} \wedge \alpha=\alpha^{\prime}$ and $\left\langle\alpha^{\prime}, \alpha\right\rangle=\left\langle\alpha^{\prime}, \alpha^{\prime}\right\rangle$. On the other hand also, $\left.\left.\mid \omega_{1}\right\lrcorner\left.\alpha\right|^{2}=\mid \omega_{1}\right\lrcorner\left.\omega_{1} \wedge \bar{\omega}_{1} \wedge \gamma\right|^{2}=$ $\left|\omega_{1} \wedge \gamma\right|^{2}=\left|\bar{\omega}_{1} \wedge \omega_{1} \wedge \gamma\right|^{2}=\left|\alpha^{\prime}\right|^{2}$ since $\bar{\omega}_{1} \wedge \gamma$ does not contain $\omega_{1}$. This proves the lemma.

Proof of Lemma 5. We may assume that $\psi$ has support in some small neighborhood of a boundary point and we let $\alpha$ be a $(2 n-1)$-form such that $d V=d \rho \wedge \alpha /|d \rho|$ there. Then

$$
\begin{aligned}
t \int_{D}( & -\rho)^{t-1} \psi d V=t \int_{D}(-\rho)^{t-1} d \rho \wedge \psi \alpha /|d \rho| \\
& =-\int_{D} d(-\rho)^{t} \wedge \psi \alpha /|d \rho|=\int_{D}(-\rho)^{t} \wedge d(\psi \alpha /|d \rho|) \\
& \rightarrow \int_{D} d(\psi \alpha /|d \rho|)=\int_{\partial D} \psi \alpha /|d \rho|=\int_{\partial D} \psi d S /|d \rho|
\end{aligned}
$$

where we have used Stokes' theorem twice.

\section{A SOLUTION OF The $\bar{\partial}_{b}$-EQUATION}

In this paragraph $D=\{\rho<0\}$ is pseudoconvex and $\rho$ is a $C^{2}$ plurisubharmonic defining function. Suppose that the hermitian operator (see [8])

$$
A=i(-\rho) \boldsymbol{\Theta} \Lambda+i \partial \bar{\partial} \rho \Lambda
$$

is semipositive on $E$-valued $(n, q+1)$-forms, i.e.

$$
\langle A \alpha, \alpha\rangle \geq 0, \quad \alpha \in \mathscr{E}_{n, q+1}(\bar{D}, E) .
$$


Then Proposition 6 in $\S 3$ provides the estimate

$$
\left.\int_{D}\langle A \alpha, \alpha\rangle d V \leq \int_{\partial D} \mid \partial \rho\right\lrcorner\left.\alpha\right|^{2} d S /|d \rho|
$$

for $\alpha \in \mathscr{E}_{p, q+1}(\bar{D}, E)$ such that $D^{\prime \prime} \alpha=\left(D^{\prime \prime}\right)^{*} \alpha=0$.

If $f \in \mathscr{E}_{p, q+1}(\bar{D}, E)$ is $\bar{\partial}$-closed, we thus get

$$
\left.\left|\int_{D}\langle f, \alpha\rangle d V\right|^{2} \leq \int_{D}\left\langle A^{-1} f, f\right\rangle d V \int_{\partial D} \mid \partial \rho\right\lrcorner\left.\alpha\right|^{2} d S /|d \rho|
$$

for $\alpha \in \mathscr{E}_{p, q+1}(\bar{D}, E)$ such that $D^{\prime \prime} \alpha=\left(D^{\prime \prime}\right)^{*} \alpha=0$. In order to ease the condition that $D^{\prime \prime} \alpha=0$ in (1), we first, for simplicity, assume that $\partial D$ is strictly pseudoconvex. Then, $|\alpha|^{2} \leq C\langle i \partial \bar{\partial} \rho \Lambda \alpha, \alpha\rangle$, for $\alpha$ such that $\left.\partial \rho\right\lrcorner \alpha=$ 0 on $\partial D$ and since $|\langle\Theta \Lambda \alpha, \alpha\rangle| \leq C|\alpha|^{2}$ (recall that $E$ is assumed to be a bundle over $X$ ) we get from Proposition 7 the Basic Estimate

$$
\begin{aligned}
& \int_{D}\left|\left(D^{\prime}\right)^{*} \alpha\right|^{2} d V+\int_{\partial D}|\alpha|^{2} d S \\
& \quad \leq C\left[\int_{D}\left|D^{\prime \prime} \alpha\right|^{2} d V+\int_{D}\left|\left(D^{\prime \prime}\right)^{*} \alpha\right|^{2} d V+\int_{D}|\alpha|^{2} d V\right]
\end{aligned}
$$

if $\partial \rho-\left.\alpha\right|_{\partial D}=0$. This ensures, see [6], regularity for the $D^{\prime \prime}$-Neumann problem and then any $\alpha \in \mathscr{E}_{n, q+1}(\bar{D}, E)$ has a smooth orthogonal decomposition $\alpha=$ $\alpha^{\prime}+\alpha^{\prime \prime}$ where $\bar{\partial} \alpha^{\prime}=0$ and $\alpha^{\prime \prime}$ is orthogonal to $\bar{\partial}$-closed $E$-valued forms. In particular, $\left(D^{\prime \prime}\right)^{*} \alpha^{\prime \prime}=0$ and $\partial \rho-\left.\alpha^{\prime \prime}\right|_{\partial D}=0$, cf. the remark after Proposition 1. Thus, if $\left(D^{\prime \prime}\right)^{*} \alpha=0$ then $\left(D^{\prime \prime}\right)^{*} \alpha^{\prime}=D^{\prime \prime} \alpha^{\prime}=0$ so (1) applies to $\alpha^{\prime}$ and we hence obtain (1) for $\alpha$ as well. If $\partial D$ is just pseudoconvex, we can still decompose $\alpha=\alpha^{\prime}+\alpha^{\prime \prime}$ as before. Since then $\alpha^{\prime \prime} \in \operatorname{Dom}\left(D^{\prime \prime}\right)^{*} \cap \operatorname{Dom} D^{\prime \prime}$ (in the densely defined operator sense) there are, by Proposition 2.1.1 in [9], $\alpha_{j}^{\prime \prime} \in \mathscr{E}_{n, q}(\bar{D}, E) \cap \operatorname{Dom}\left(D^{\prime \prime}\right)^{*}$ such that $\alpha_{j}^{\prime \prime} \rightarrow \alpha^{\prime \prime}$ in graph norm. In particular $\partial \rho-\left.\alpha_{j}^{\prime \prime}\right|_{\partial D}=0$. If $\alpha_{j}^{\prime}=\alpha-\alpha_{j}^{\prime \prime}$, then $\alpha_{j}^{\prime} \rightarrow \alpha^{\prime}$ in graph norm and $\left.\partial \rho\right\lrcorner \alpha_{\partial D}=$ $\partial \rho\lrcorner\left.\alpha_{j}^{\prime}\right|_{\partial D}$. Since also $\left(D^{\prime \prime}\right)^{*} \alpha_{J}^{\prime \prime} \rightarrow 0, D^{\prime \prime} \alpha_{j}^{\prime \prime} \rightarrow 0$ and $\int\left\langle f, \alpha_{j}^{\prime}\right\rangle \rightarrow \int\langle f, \alpha\rangle$ one can proceed as before, but instead using the variant of (11) in which $\left(D^{\prime \prime}\right)^{*} \alpha$ and $D^{\prime \prime} \alpha$ are not supposed to vanish, cf. (10). By Proposition 2 we then have proved

Theorem 2. Let $E \rightarrow X$ be a hermitian holomorphic vector bundle over the Kähler manifold $X$, and let $D=\{\rho<0\}$ be a pseudoconvex relatively compact domain and $\rho$ a $C^{2}$ plurisubharmonic defining function. Also suppose that $A=i(-\rho) \boldsymbol{\theta} \Lambda+i \partial \bar{\partial} \rho \Lambda$ is semipositive on E-valued $(n, q+1)$-forms. If $f \in$ $\mathscr{E}_{n, q+1}(\bar{D}, E)$ is $D^{\prime \prime}$-closed, then there is a solution to $\bar{\partial}_{b} u=f$ in $L_{n, q}^{2}(\partial D, E)$ such that

$$
\left.\int_{\partial D} \mid \partial \rho\right\lrcorner\left. u\right|^{2} d S /|\partial \rho|^{3} \leq \int_{D}\left\langle A^{-1} f, f\right\rangle d V
$$

We recall that a bundle $E$ is called Nakano semipositive if $\langle\Theta \Lambda \alpha, \alpha\rangle \geq 0$ for all $\alpha \in \mathscr{E}_{n, 1}(X, E)$.

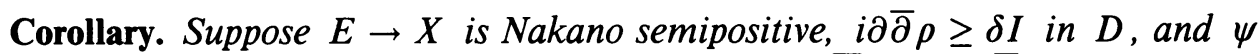
is smooth and plurisubharmonic. Then if $f \in \mathscr{E}_{n, 1}(\bar{D}, E)$ is $\bar{\partial}$-closed, there is 
a solution $u$ to $\bar{\partial}_{b} u=f$ such that

$$
\int_{\partial D}|u|^{2} e^{-\psi} d S /|d \rho| \leq \frac{1}{\delta} \int_{D}|f|^{2} e^{-\psi} d V .
$$

In particular, one can let $E$ be the trivial bundle over a domain $D$ in $\mathbb{C}^{n}$ and thus get $(2)$ for $(0,1)$-forms $f$.

\section{THE DIVISION PROBLEM}

When proving Theorem 1, we assume that $q$ and $g$ are holomorphic in a neighborhood of $\bar{D}$. The general case then follows by a normal family argument on the solutions in $D_{\varepsilon}=\{\rho<-\varepsilon\}$, since it turns out that the occurring constants only depend on derivatives up to second order of $\rho$ near $\partial D$.

Remark. It is proved in [4] that any $C^{2}$ pseudoconvex domain admits a $C^{2}$ defining function $\rho$ such that $-(-\rho)^{\eta}$ is (strictly) plurisubharmonic in $D$ for some $\eta>0$. Unfortunately, by our method the constants belonging to $D_{\varepsilon}=$ $\{\rho<-\varepsilon\}$ seem to be unbounded when $\varepsilon \rightarrow 0$ so we cannot prove Theorem 1 in this general case.

We thus have to consider the following situation. An exact sequence $0 \rightarrow$ $S \stackrel{j}{\rightarrow} E \stackrel{g}{\rightarrow} Q \rightarrow 0$ of hermitian holomorphic vector bundles over $X$, such that $j$ and $g$ are holomorphic, $S$ is equipped with the metric induced from $E$, $|g| \leq 1$ and det $g g^{*} \geq \delta^{2}>0$. The problem then is if for any holomorphic $q \in \mathscr{E}_{n, 0}(\bar{D}, Q)$ there is a holomorphic solution $u \in \mathscr{E}_{n, 0}(D, S)$ to

$$
g u=q
$$

such that

$$
\int_{\partial D}|u|^{2} d S \leq C^{2} \int_{\partial D}|q|^{2} d S
$$

To find such holomorphic solutions, we proceed as follows. First we note that the pointwise minimal solution $\gamma q=g^{*}\left(g g^{*}\right)^{-1} q$ satisfies (2). Moreover, $\bar{\partial}(\gamma q)=(\bar{\partial} \gamma) q$ is a $\bar{\partial}$-closed $(n, 1)$-form with values in $S$, since $g \bar{\partial} \gamma q=$ $\bar{\partial}(g \gamma q)=\bar{\partial} q=0$. The hard step then is to find a $v \in L_{n, 0}^{2}(\partial D, S)$ satisfying (2) such that $\bar{\partial}_{b} v=(\bar{\partial} \gamma) q$ in $S$, i.e. such that

$$
\left.\int_{\partial D}\langle v, \partial \rho\lrcorner \xi\right\rangle d S /|d \rho|=\int_{D}\langle(\bar{\partial} \gamma) q, \xi\rangle d V
$$

for all $\xi \in \mathscr{E}_{n, 1}(\bar{D}, S)$ such that $\left(D^{\prime \prime}\right)_{S}^{*} \xi=0$.

We now claim that (3) implies that actually $\bar{\partial}_{b} v=(\bar{\partial} \gamma) q$ in $E$. Taking this for granted for the moment we conclude that $\bar{\partial}_{b}(\gamma q-v)=0$ in (the trivial bundle) $E$ which means that $u=\gamma q-v$ satisfies the tangential CauchyRiemann equation weakly.

This implies that $u$ is the boundary values of a $U \in H^{2}(D)$ with norm

$$
\|U\|_{H^{2}}^{2}=\int_{\partial D}|u|^{2} d S=\int_{\partial D}|v|^{2} d S+\int_{\partial D}|\gamma q|^{2} d S
$$

( $v$ and $\gamma q$ being orthogonal) and since $g U=q$ on $\partial D$, it must hold in $D$. 
Thus our problem is solvable if (and only if) we can obtain (3) such that the $L^{2}(\partial D)$-norm of $v$ is controlled by $\left(\int_{\partial D}|q|^{2} d S\right)^{1 / 2}$. By Proposition 2 this amounts to verify the inequality

$$
\left.\left|\int_{D}\langle\bar{\partial} \gamma q, \xi\rangle d V\right|^{2} \leq C_{\delta}^{2} \int_{\partial D}|q|^{2} d S \int_{\partial D} \mid \partial \rho\right\lrcorner\left.\xi\right|^{2} d S /|\partial \rho|^{2}
$$

for all $\xi \in \mathscr{E}_{n, 1}(\bar{D}, S)$ such that $\left(D^{\prime \prime}\right)^{*} \xi=0$.

When trying to prove the estimate (4) one encounters two main difficulties. Firstly, although $E$ and $Q$ are trivial bundles with trivial metrics in our case, $S$ acquires negative curvature which must be taken care of. However, it turns out that the curvature on $S$ becomes nonnegative if the original metric is modified by a factor $e^{-\varphi}$, where $\varphi$ is a bounded plurisubharmonic function. Since $e^{\varphi}$ is bounded, it does not affect the estimates in any essential way. Secondly, even if we forget about the curvature problems, i.e. consider the scalar-valued case, an essential difficulty remains. As was mentioned in the introduction, one cannot use Theorem 2 directly since we must use more information about the righthand side in our $\bar{\partial}_{b}$-equation than just a size estimate. Here the Wolff trick comes into play. Restricted to the scalar-valued case, our "Wolff theorem" can be stated

Proposition 8. Suppose $f \in \mathscr{E}_{n, 1}(\bar{D})$ is $\bar{\partial}$-closed, and that there is a bounded plurisubharmonic $\varphi$ on $\bar{D}$ such that $|\langle f, \alpha\rangle|^{2} \leq\langle i \partial \bar{\partial} \varphi \Lambda \alpha, \alpha\rangle$ and

$$
\sum_{k=1}^{n}\left|\left\langle\frac{\partial f}{\partial z_{k}}, \alpha\right\rangle\right|^{2} \leq \Delta \varphi\langle i \partial \bar{\partial} \varphi \Lambda \alpha, \alpha\rangle
$$

for all $(n, 1)$-forms $\alpha$. Then for any holomorphic $q$ there is a solution $v \in$ $L_{n, 0}^{2}(\partial D)$ to $\bar{\partial}_{b} v=f q$ such that

$$
\int_{\partial D}|v|^{2} d S \leq C^{2} \int_{\partial D}|q|^{2} d S
$$

where $C$ only depends on $\|\varphi\|_{\infty}$ and $D$.

This proposition will be proved implicitly in the next paragraph. In the unit disc the assumption in the proposition is essentially, see [2], that $\left(1-|\zeta|^{2}\right)|f|^{2}$ and $\left(1-|\zeta|^{2}\right)|\partial f / \partial z|$ be Carleson measures, and the conclusion of the proposition implies, cf. the introduction, that there is a bounded solution.

Proof of the claim above. We actually have to verify that if $v \in L_{n, 0}^{2}(\partial D, S)$ and (3) holds for all $\xi \in \mathscr{E}_{n, 1}(\bar{D}, S)$ such that $\left(D^{\prime \prime}\right)_{S}^{*} \xi=0$, then it also holds for all $\alpha \in \mathscr{E}_{n, 1}(\bar{D}, E)$ such that $\left(D^{\prime \prime}\right)_{E}^{*} \alpha=0$. However, if $p: E \rightarrow S$ is the orthogonal projection, then clearly (3) holds for $\xi=\alpha-p \alpha$. Moreover, if $\left(D^{\prime \prime}\right)_{E}^{*} \alpha=0$, then for any compactly supported $\eta \in \mathscr{E}_{n, 1}(\bar{D}, S)$,

$$
0=\int_{D}\left\langle\left(D^{\prime \prime}\right)_{E}^{*} \alpha, \eta\right\rangle=\int_{D}\langle\alpha, \bar{\partial} \eta\rangle=\int_{D}\langle p \alpha, \bar{\partial} \eta\rangle=\int_{D}\left\langle\left(D^{\prime \prime}\right)_{S}^{*} p \alpha, \eta\right\rangle,
$$

so that $\left(D^{\prime \prime}\right)_{S}^{*} p \alpha=0$. Hence (3) holds for $\alpha=p \alpha+(\alpha-p \alpha)$. 


\section{Proofs of Theorems 1 AND $1^{\prime}$}

Let $\beta$ be the element in $\mathscr{E}_{1,0}(\bar{D}, \operatorname{Hom}(S, Q))$ such that its adjoint, with respect to the quotient metric on $Q, \beta^{*} \in \mathscr{E}_{0,1}(\bar{D}, \operatorname{Hom}(Q, S))$ equals $-\bar{\partial} \gamma$. Thus our equation to be solved ((3) in $\S 5)$ becomes $\bar{\partial}_{b} v=-\beta^{*} q$. Moreover, since $E$ has no curvature,

$$
\boldsymbol{\Theta}_{S}=\beta^{*} \wedge \beta
$$

and since $Q$ has no curvature,

$$
-i\left\langle\beta^{*} \wedge \beta \Lambda \xi, \xi\right\rangle \leq r\langle i \partial \bar{\partial} \psi \Lambda \xi, \xi\rangle, \quad \xi \in \mathscr{E}_{n, 1}(\bar{D}, S),
$$

where $r=\min (n, \operatorname{rank} S)$ and $\psi=\log \operatorname{det} g g^{*}$ (note that $g^{*}$ depends on the metric on $Q)$. We also need the estimate

$$
\left|\left\langle\beta^{*} q, \xi\right\rangle\right|^{2} \leq-\left|g^{*}\left(g g^{*}\right)^{-1} q\right|^{2} i\left\langle\beta^{*} \wedge \beta \Lambda \xi, \xi\right\rangle
$$

for $q \in \mathscr{E}_{n, 0}(\bar{D}, Q)$ and $\xi \in \mathscr{E}_{n, 1}(\bar{D}, S)$. For proofs of (1), (2) and (3) we refer to [12].

Recall (Proposition 6 in $\S 3$ ) that

$$
\begin{gathered}
\int_{D}(-\rho)\left\langle i\left(\Theta_{S}+\partial \bar{\partial} \varphi\right) \Lambda \alpha, \alpha\right\rangle e^{-\varphi} d V+\int_{D}\langle i \partial \bar{\partial} \rho \Lambda \alpha, \alpha\rangle e^{-\varphi} d V \\
\left.+\int_{D}(-\rho)\left|\left(D^{\prime}\right)^{*} \alpha\right|^{2} e^{-\varphi} d V=\int_{\partial D} \mid \partial \rho\right\lrcorner\left.\alpha\right|^{2} e^{-\varphi} d S /|d \rho|
\end{gathered}
$$

for $\alpha \in \mathscr{E}_{n, 1}(\bar{D}, S)$ if $\bar{\partial} \alpha=\left(D^{\prime \prime}\right)^{* \varphi} \alpha=0$.

Hence if $\varphi=(r+\varepsilon) \psi$ and $\rho$ is plurisubharmonic, we get by (1) and (2)

$$
\left.\int_{D}(-\rho)\left|\left(D^{\prime}\right)^{*} \alpha\right|^{2} e^{-\varphi} d V \leq \int_{\partial D} \mid \partial \rho\right\lrcorner\left.\alpha\right|^{2} e^{-\varphi} d S /|d \rho|
$$

and

$$
\left.\int_{D}(-\rho)\langle i \partial \bar{\partial} \varphi \Lambda \alpha, \alpha\rangle e^{-\varphi} d V \leq \frac{r+\varepsilon}{r} \int_{\partial D} \mid \partial \rho\right\lrcorner\left.\alpha\right|^{2} e^{-\varphi} d S /|d \rho| .
$$

From (3) we also get

$$
\left|\left\langle\beta^{*} q, \xi\right\rangle\right|^{2} \leq\left|g^{*}\left(g g^{*}\right)^{-1} q\right|^{2} \frac{r}{r+\varepsilon}\langle i \partial \bar{\partial} \varphi \Lambda \xi, \xi\rangle,
$$

for $\xi \in \mathscr{E}_{n, 1}(\bar{D}, S)$ and $q \in \mathscr{E}_{n, 0}(\bar{D}, Q)$. We now claim that Theorem 1 follows from

Proposition 9. If $D$ and $\rho$ are as in Theorem 1 , then

$$
\left.\left|\int_{D}\left\langle\beta^{*} q, \xi\right\rangle d V\right|^{2} \leq C_{\delta}^{2} \int_{\partial D}|q|^{2} d S \int_{\partial D} \mid \partial \rho\right\lrcorner\left.\xi\right|^{2} e^{\varphi} d S
$$

for any holomorphic $q \in \mathscr{E}_{n, 0}(\bar{D}, Q)$ and $\xi \in \mathscr{E}_{n, 1}(\bar{D}, S)$ such that $\left(D^{\prime \prime}\right)^{* \varphi}\left(e^{q} \xi\right)$ $=\bar{\partial}\left(e^{\varphi} \xi\right)=0$.

Here $C_{\delta}$ is the constant described in Theorem 1.

Proof of Theorem 1. By the discussion in $\S 5$ it is enough to verify (4) in $\S 5$ for $\xi \in \mathscr{E}_{n, 1}(\bar{D}, S)$ such that $\left(D^{\prime \prime}\right)^{*} \xi=0$. Note that

$$
\left(D^{\prime \prime}\right)^{*} \xi=0 \text { iff }\left(D^{\prime \prime}\right)^{* \varphi}\left(e^{\varphi} \xi\right)=0 .
$$

Putting $\alpha=e^{\varphi} \xi$, (7) then says that 


$$
\left.\left|\int_{D}\left\langle\beta^{*} q, \alpha\right\rangle e^{-\varphi} d V\right|^{2} \leq C_{\delta}^{2} \int_{\partial D}|q|^{2} d S \int_{\partial D} \mid \partial \rho\right\lrcorner\left.\alpha\right|^{2} e^{-\varphi} d S
$$

for all $\alpha$ such that $\bar{\partial} \alpha=\left(D^{\prime \prime}\right)^{* \varphi} \alpha=0$. As in $\S 4$, we can obtain (8) for all $\alpha$ with $\left(D^{\prime \prime}\right)^{* \varphi} \alpha=0$. But this means that (7) holds for all $\xi$ with $\left(D^{\prime \prime}\right)^{*} \xi=0$. Finally $e^{\varphi}=\left(\operatorname{det} g g^{*}\right)^{r+\varepsilon} \leq 1$ by assumption, and hence we have verified (4) in $\S 5$.

Proof of Proposition 9. We consider $g$ as a $j \times k$-matrix of holomorphic functions on $\bar{D}$ and use the norms

$$
\|g\|^{2}=\sum_{\tau \nu}\left|g_{\tau \nu}\right|^{2}, \quad\left|g^{\prime}\right|^{2}=\sum_{i \tau \nu}\left|\partial g_{\tau \nu} / \partial \zeta_{i}\right|^{2}
$$

The assumptions on $g$ imply that

$$
\left|\left(g g^{*}\right)^{-1}\right| \lesssim 1 / \delta^{2},\left|g^{*}\left(g g^{*}\right)^{-1} q\right|^{2} \lesssim\left(1 / \delta^{2}\right)|q|^{2}
$$

If $\Delta$ is the $\mathbb{R}^{2 n}$-Laplacian, then

$$
\left|g^{\prime}\right|^{2} /|g|^{2-2 \varepsilon} \leq C_{\varepsilon} \Delta|g|^{2 \varepsilon}, \quad \varepsilon>0 \text {. }
$$

We also need the inequalities

$$
\int_{D}(-\rho)\left|f^{\prime}\right|^{2} d V \lesssim \int_{\partial D}|f|^{2} d S
$$

and

$$
\int_{D}(-\rho)|f|^{2} \Delta \psi e^{\psi} d V \lesssim \int_{\partial D}|f|^{2} e^{\psi} d S
$$

for holomorphic $f$ and subharmonic $\psi$. They follow from Green's formula. Recall that $-\beta^{*}=\bar{\partial} \gamma=\bar{\partial}\left[g\left(g g^{*}\right)^{-1}\right]$ so that, for an $S$-valued $\xi$,

$$
-\left\langle\beta^{*} q, \xi\right\rangle=\left\langle(\partial g)^{*}\left(g g^{*}\right)^{-1} q, \xi\right\rangle \text {. }
$$

We have to estimate

$$
I=\int_{D}\left\langle\beta^{*} q, \xi\right\rangle d \lambda,\left(D^{\prime \prime}\right)^{* \varphi}\left(e^{\varphi} \xi\right)=\bar{\partial}\left(e^{\varphi} \xi\right)=0 .
$$

Let $\chi$ be a smooth function which is identically 1 near $\partial D$ and such that $\partial \rho$ is nonvanishing on its support. If $L=\chi|\partial \rho|^{-2} \sum_{1}^{n}\left(\partial \rho / \partial \bar{\zeta}_{j}\right) \partial / \partial \zeta_{j}$, then we can write

$$
I=\int_{D}(1-\chi)\left\langle\beta^{*} q, \xi\right\rangle d V-\int_{D} L(-\rho)\left\langle\beta^{*} q, \xi\right\rangle d V,
$$

and an integration by parts in the second integral gives us

$$
\begin{aligned}
I= & \int_{D}(1-\chi)\left\langle\beta^{*} q, \xi\right\rangle d V+\int_{D}(-\rho) O(1)\left\langle\beta^{*} q, \xi\right\rangle d V \\
& +\int_{D}(-\rho)\left\langle\left(L \beta^{*}\right) q, \xi\right\rangle d V+\int_{D}(-\rho)\left\langle\beta^{*} L q, \xi\right\rangle d V \\
& +\int_{D}(-\rho)\left\langle\beta^{*} q,(\bar{L} \varphi) \xi+\bar{L} \xi\right\rangle d V-\int_{D}(-\rho)\left\langle\beta^{*} q, \bar{L} \varphi \xi\right\rangle d V \\
= & \mathrm{I}_{0}+\mathrm{I}_{1}+\mathrm{I}_{2}+\mathrm{I}_{3}+\mathrm{I}_{4}+\mathrm{I}_{5}
\end{aligned}
$$


where $O(1)$ only depends on derivatives up to second order of $\rho$ and $\chi$, and where $\varphi=(r+\varepsilon) \log \operatorname{det} g g^{*}$. The proof is concluded by estimating each $\mathrm{I}_{i}$. By (6) and Schwarz inequality we have that, for $i=0,1$,

$$
\left|\mathbf{I}_{i}\right|^{2} \lesssim \int_{D}(-\rho)\left|g^{*}\left(g g^{*}\right)^{-1} q\right|^{2} e^{-\varphi} d V \int_{D}(-\rho)\langle i \partial \bar{\partial} \varphi \Lambda \xi, \xi\rangle e^{\varphi} d V
$$

Now, cf. (9),

$$
\begin{aligned}
\int_{D}(-\rho)\left|g^{*}\left(g g^{*}\right)^{-1} q\right|^{2} e^{-\varphi} d V & \lesssim \frac{1}{\delta^{2} \delta^{2(r+\varepsilon)}} \int_{D}(-\rho)|q|^{2} d V \\
& \lesssim\left(\frac{1}{\delta^{1+r+\varepsilon}}\right)^{2} \int_{\partial D}|q|^{2} d S
\end{aligned}
$$

since $q$ is holomorphic (cf. (12) with e.g. $\psi=|\zeta|^{2}$ ). Also

$$
\int_{D}(-\rho)\langle i \partial \bar{\partial} \varphi \Lambda \xi, \xi\rangle e^{\varphi} d V=\int_{D}(-\rho)\left\langle i \partial \bar{\partial} \varphi \Lambda\left(e^{\varphi} \xi\right), e^{\varphi} \xi\right\rangle e^{-\varphi} d V
$$

and hence by $\left.(5), \lesssim \int_{\partial D} \mid \partial \rho\right\lrcorner\left.\xi\right|^{2} e^{\varphi} d S$. Thus we have obtained the required estimate for $I_{0}$ and $I_{1}$. To handle $I_{2}$, first note that

$$
\begin{aligned}
-\left\langle\left(L \beta^{*}\right) q, \xi\right\rangle & =\left\langle(\partial g)^{*}\left(g g^{*}\right)^{-1}(L g) g^{*}\left(g g^{*}\right)^{-1} q, \xi\right\rangle \\
& =\left\langle\beta^{*}(L g) g^{*}\left(g g^{*}\right)^{-1} q, \xi\right\rangle
\end{aligned}
$$

so by (6) and (9),

$$
\begin{aligned}
\left|\left\langle\left(L \beta^{*}\right) q, \xi\right\rangle\right|^{2} & \leq\left|g^{*}\left(g g^{*}\right)^{-1}(L g) g^{*}\left(g g^{*}\right)^{-1} q\right|^{2} e^{-\varphi}\langle i \partial \bar{\partial} \varphi \Lambda \xi, \xi\rangle e^{\varphi} \\
& \lesssim \frac{1}{\delta^{2} \delta^{2(r+\varepsilon)} \delta^{2 \varepsilon}} \frac{\left|g^{\prime}\right|^{2}}{\left(\operatorname{det} g g^{*}\right)^{1-\varepsilon}}|q|^{2}\langle i \partial \bar{\partial} \varphi \Lambda \xi, \xi\rangle e^{\varphi}
\end{aligned}
$$

If $g$ is a row matrix, i.e. $j=1$, then $\operatorname{det} g g^{*}=|g|^{2}$ so we can use (10) and get

$$
\begin{aligned}
\left|\mathbf{I}_{2}\right|^{2} & \leq\left(\frac{1}{\delta^{1+r+2 \varepsilon}}\right)^{2} \int_{D}(-\rho)|q|^{2} \Delta|g|^{2 \varepsilon} e^{|g|^{2 \varepsilon}} \int_{D}(-\rho)\langle i \partial \bar{\partial} \varphi \Lambda \xi, \xi\rangle e^{\varphi} \\
& \left.\lesssim\left(\frac{1}{\delta^{1+r+2 \varepsilon}}\right)^{2} \int_{\partial D}|q|^{2} d S \int_{\partial D} \mid \partial \rho\right\lrcorner\left.\xi\right|^{2} e^{\varphi} d S .
\end{aligned}
$$

If $j>1$ we estimate $1 / \operatorname{det} g g^{*}$ by $1 / \delta^{2}$ and use the simpler inequality $\left|g^{\prime}\right|^{2} \leq$ $\Delta|g|^{2}$. For simplicity we assume for the rest of the proof that $j=1$. To handle $\mathrm{I}_{3}$, we note that

$$
\left|\left\langle\beta^{*} L q, \xi\right\rangle\right|^{2} \lesssim\left(\frac{1}{\delta^{1+r+\varepsilon}}\right)^{2}\left|q^{\prime}\right|^{2}\langle i \partial \bar{\partial} \varphi \Lambda \xi, \xi\rangle e^{\varphi}
$$

and here the first factor is treated by (11) and the second one as before. Further, we have

$$
\begin{aligned}
\left|\mathrm{I}_{4}\right|^{2} & \lesssim \int_{D}(-\rho)\left|\beta^{*} q\right|^{2} e^{-\varphi} d V \int_{D}(-\rho)|(\bar{L} \varphi) \xi+\bar{L} \xi|^{2} e^{\varphi} d V \\
& \lesssim\left(\frac{1}{\delta^{1+r+2 \varepsilon}}\right)^{2} \int_{D}(-\rho) \frac{\left|g^{\prime}\right|^{2}}{|g|^{2-2 \varepsilon}}|q|^{2} d V \int_{D}(-\rho)\left|\bar{L}\left(e^{\varphi} \xi\right)\right|^{2} e^{-\varphi} d V
\end{aligned}
$$


The first factor is handled as before and the second one is estimated by (4). Finally,

$$
\left|\mathbf{I}_{5}\right|^{2} \lesssim\left(\frac{1}{\delta^{1+r+\varepsilon}}\right)^{2} \int_{D}(-\rho)|\bar{L} \varphi|^{2}|q|^{2} d V \int_{D}(-\rho)\langle i \partial \bar{\partial} \varphi \Lambda \xi, \xi\rangle e^{\varphi} d V .
$$

Note that $|\bar{L} \varphi|^{2} \leq\left|g^{\prime}\right|^{2} /|g|^{2}$ so that the first factor is dominated by

$$
\frac{1}{\delta^{2 \varepsilon}} \int_{D}(-\rho)\left(\Delta|g|^{2 \varepsilon}\right)|q|^{2} e^{|g|^{2 \varepsilon}} d V \lesssim \frac{1}{\delta^{2 \varepsilon}} \int_{\partial D}|q|^{2} d S
$$

and hence the proposition is proved.

Proof of Theorem $1^{\prime}$. It is enough to show that

$$
\left.\left|\int_{D}\left\langle\beta^{*} q, \xi\right\rangle d V\right|^{2} \leq C_{\varepsilon} \int_{\partial D} e^{-\chi} d S \int_{\partial D} \mid \partial \rho\right\lrcorner\left.\xi\right|^{2} e^{\varphi+\chi}
$$

for all $\xi$ such that $\left(D^{\prime \prime}\right)^{* \psi}\left(e^{\psi} \xi\right)=\bar{\partial}\left(e^{\psi} \xi\right)=0$, where we have put $\psi=\varphi+\chi=$ $\left(r+\varepsilon^{\prime}\right) \log |g|^{2}+\chi$ and $\varepsilon^{\prime}$ is less than the $\varepsilon$ in the hypothesis of Theorem $1^{\prime}$.

Then most arguments when estimating the left-hand side work as before. We have just two new difficulties. For the term $I_{3}$ we have

$$
\left|\mathrm{I}_{3}\right|^{2} \lesssim \int_{D}(-\rho)|L q|^{2} /|g|^{2\left(1+r+\varepsilon^{\prime}\right)} e^{-\chi} \int_{D}(-\rho)\langle i \partial \bar{\partial} \psi \Lambda \xi, \xi\rangle e^{\psi} .
$$

Since $|g|^{2(1+r+\varepsilon)} \geq|q|^{2},|g|^{2\left(1+r+\varepsilon^{\prime}\right)} \geq|q|^{2-\varepsilon^{\prime \prime}}$ and hence the first factor is

$$
\begin{aligned}
& \lesssim \int_{D}(-\rho) \Delta|q|^{\varepsilon^{\prime \prime}} e^{-\chi} \leq \int_{D}(-\rho) \Delta\left(|q|^{2^{\prime \prime}}-\chi\right) e^{|q|^{e^{\prime \prime}-\chi}} \\
& \lesssim \int_{\partial D} e^{|q|^{\varepsilon^{\prime \prime}-\chi}} d S \lesssim \int_{\partial D} e^{-\chi} d S .
\end{aligned}
$$

When estimating $I_{5}$ we show up with a factor

$$
\begin{aligned}
& \int_{D}(-\rho)|q|^{2}|\nabla \psi|^{2} e^{-\psi} \leq \int_{D}(-\rho)|g|^{\varepsilon^{\prime \prime}}|\nabla \psi|^{2} e^{-\chi} \\
& \quad \lesssim \int_{D}(-\rho)|g|^{\varepsilon^{\prime \prime}}\left|\nabla\left(\log |g|^{2}\right)\right|^{2} e^{-\chi}+\int_{D}(-\rho)|\nabla \chi|^{2} e^{-\chi}=a_{1}+a_{2} .
\end{aligned}
$$

But

$$
a_{1} \lesssim \int_{D}(-\rho) \Delta\left(|g|^{\varepsilon^{\prime \prime}}-\chi\right) e^{|g|^{e^{\prime \prime}-\chi}} \lesssim \int_{\partial D} e^{-\chi}
$$

and so is $a_{2}$. This concludes the proof of Theorem $1^{\prime}$.

\section{REFERENCES}

1. M. Andersson, The corona theorem for matrices, Math. Z. 201 (1989), 121-130.

2. B. Berndtsson, $\bar{\partial}_{b}$ and Carleson inequalities, Complex Analysis. II, (Univ. of Maryland, 1985-86), Lecture Notes in Math., vol. 1276, Springer, 1987, pp. 42-54.

3. K. Diedrich and J. E. Fornaess, Pseudoconvex domains: An example with nontrivial nebenhuelle, Math. Ann. 225 (1977), 275-292.

4. __ Pseudoconvex domains: Bounded strictly plurisubharmonic exhaustion functions, Invent. Math. 39 (1977), 129-141.

5. J. E. Fornaess and N. Sibony, Smooth pseudoconvex domains in $\mathbb{C}^{2}$ for which the corona theorem and $L^{p}$ estimates for $\bar{\partial}$ fail, preprint 1991. 
6. G. B. Folland and J. J. Kohn, The Neumann problem for the Cauchy-Riemann complex, Princeton Univ. Press, 1972.

7. T. W. Gamelin, Wolff's proof of the corona theorem, Israel J. Math. 37 (1980), 113-119.

8. P. A. Griffiths and J. Harris, Principles of algeraic geometry, Wiley-Interscience, New York, 1978.

9. L. Hörmander, $L^{2}$ estimates and existence theorems for the $\bar{\partial}$ operator, Acta Math. 113 (1965), 89-152.

10. $\ldots$, Generators for some rings of analytic functions, Bull. Amer. Math. Soc. 73 (1967), 943-949.

11. N. Sibony, Problème de la courenne pour les domaines faiblement pseudoconvexes à bord lisse, Ann. of Math. 126 (1987), 675-682.

12. H. Skoda, Morphismes surjectifs de fibres vectoriels semi-positifs, Ann. Sci. Ecole Norm. Sup. 11 (1978), 577-611.

13. N. Th. Varopoulos, BMO functions and the $\bar{\partial}$-equation, Pacific J. Math. 71 (1977), 221-273.

Department of Mathematics, Chalmers University of Technology and The UniverSITY OF GOTEBORG, S-412 96 GOTEBORG, SWEDEN

E-mail address: matsa@math.chalmers.se 